\title{
ON THE OPTIMAL STOPPING PROBLEMS WITH MONOTONE THRESHOLDS
}

\author{
MITSUSHI TAMAKI, ${ }^{*}$ Aichi University
}

\begin{abstract}
As a class of optimal stopping problems with monotone thresholds, we define the candidate-choice problem (CCP) and derive two formulae for calculating its expected payoff. We apply the first formula to a particular CCP, i.e. the best-choice duration problem treated by Ferguson et al. (1992). The recall case is also examined as a comparison. We also derive the distribution of the stopping time of the CCP and find, as a by-product, that the best-choice problem has a remarkable feature in that the optimal probability of choosing the best is just the expected value of the (proportional) stopping time. The similarity between the best-choice duration problem and the best-choice problem with uniform freeze studied by Samuel-Cahn (1996) is recognized.
\end{abstract}

Keywords: Secretary problem; best-choice problem; duration problem; best-choice duration problem; candidate-choice problem; monotone rule; planar Poisson process

2010 Mathematics Subject Classification: Primary 60G40

Secondary $62 \mathrm{~L} 15$

\section{Introduction}

As a typical optimal stopping problem with monotone thresholds, we first review the (fullinformation) best-choice problem originally studied by Gilbert and Mosteller (1966, Section 3) as a variation of the secretary problem. A known number, $n$, of objects appear one at a time. Let $X_{k}, 1 \leq k \leq n$, denote the value of the $k$ th object and suppose that $X_{1}, X_{2}, \ldots, X_{n}$ are independent and identically distributed random variables with a known continuous distribution $F$. Since $F\left(X_{k}\right)$ is uniformly distributed on the interval $(0,1)$ and order relationship among $X_{1}, X_{2}, \ldots, X_{n}$ is preserved under this monotonic transformation, we can assume without loss of generality that $X_{1}, X_{2}, \ldots, X_{n}$ are uniformly distributed on the interval $(0,1)$. As each object appears, we observe its value and decide either to select or reject it based on the values observed so far. Once an object is chosen, the process terminates. The objective of the problem is to find a stopping rule which maximizes the probability of choosing the best, i.e. stopping with the largest of $X_{1}, X_{2}, \ldots, X_{n}$ and compute the probability of choosing the best under an optimal stopping rule (the term 'stopping' is usually identified with selection in the secretary problem).

Let $L_{k}=\max \left(X_{1}, \ldots, X_{k}\right), 1 \leq k \leq n$, and call the $k$ th object (or $X_{k}$ ) candidate if it is a relative maximum, i.e. $X_{k}=L_{k}$. Obviously an optimal stopping rule of the best-choice problem only stops with a candidate except for the last stage (if we pass over the first $n-1$ objects, we stop with the last object). Consider now a class of stopping rules of the form

$$
\tau_{n}=\tau_{n}(a)=\min \left\{k: X_{k} \geq a_{k}, X_{k}=L_{k}\right\} \wedge n,
$$

Received 2 January 2014; revision received 17 December 2014.

* Postal address: Department of Business Administration, Aichi University, Nagoya Campus, Hiraike 4-60-6, Nakamura, Nagoya, Aichi, 453-8777, Japan. Email address: tamaki@vega.aichi-u.ac.jp 
where $\boldsymbol{a}=\left(a_{1}, a_{2}, \ldots, a_{n}\right)$ is a given sequence of thresholds satisfying the monotone condition $1 \geq a_{1} \geq a_{2} \geq \cdots \geq a_{n} \geq 0$. This rule is simply referred to as a monotone rule (with thresholds $\boldsymbol{a}$ ).

Gilbert and Mosteller (1966, Theorem 4) showed that the probability of choosing the best object under a monotone rule $\tau_{n}(\boldsymbol{a})$ is calculated as

$$
v_{n}(\boldsymbol{a})=\frac{1-a_{1}^{n}}{n}+\sum_{j=1}^{n-1}\left[\sum_{k=1}^{j} \frac{a_{k}^{j}}{j(n-j)}-\sum_{k=1}^{j} \frac{a_{k}^{n}}{n(n-j)}-\frac{a_{j+1}^{n}}{n}\right]
$$

and that the optimal stopping rule is within the class of monotone rules and the particular thresholds $\boldsymbol{a}^{*}=\left(a_{1}^{*}, a_{2}^{*}, \ldots, a_{n}^{*}\right)$ specifies the optimal stopping rule if $a_{n}^{*}=0$ and $a_{k}^{*}, k<n$, is a unique root $x \in(0,1)$ of the equation

$$
\sum_{j=1}^{n-k} \frac{1}{j}\left(x^{-j}-1\right)=1
$$

Taking this condition into consideration, Sakaguchi (1973) gave a neat expression

$$
v_{n}^{*}=v_{n}\left(\boldsymbol{a}^{*}\right)=\frac{1}{n}\left[1+\sum_{k=1}^{n-1} \sum_{j=k}^{n-1} \frac{\left(a_{k}^{*}\right)^{j}}{j}\right]
$$

for the optimal probability (to be exact, (1.3) was obtained by Sakaguchi as a simpler form of the equivalent equation first obtained by Gilbert and Mosteller). As will be shown in Section 2, the best-choice problem has a remarkable feature in that the optimal probability is just the expected value of the optimal (proportional) stopping time, i.e.

$$
v_{n}^{*}=\mathbb{E}\left[\frac{\tau_{n}^{*}}{n}\right]
$$

where $\tau_{n}^{*}=\tau_{n}\left(\boldsymbol{a}^{*}\right)$. To the best of the author's knowledge, this result is new.

If we introduce the exponential-integral functions

$$
I(c)=\int_{1}^{\infty} \frac{\mathrm{e}^{-c x}}{x} \mathrm{~d} x, \quad J(c)=\int_{0}^{1} \frac{\mathrm{e}^{c x}-1}{x} \mathrm{~d} x
$$

and define $c^{*}(\approx 0.80435)$ as a solution $c$ to the equation $J(c)=1$, the limiting optimal probability is given by Samuels (1982) as

$$
v^{*}=\lim _{n \rightarrow \infty} v_{n}^{*}=\mathrm{e}^{-c^{*}}+\left(\mathrm{e}^{c^{*}}-c^{*}-1\right) I\left(c^{*}\right) \approx 0.580164
$$

Thus far we have shown the various aspects of the best-choice problem. Besides the bestchoice problem, there are many optimal stopping problems having the property that the selection is restricted to a candidate except for the last stage and the optimal rule falls within the class of monotone rules. The problem having such a property is henceforth referred to as a candidatechoice problem (CCP). See Section 2 for more details of the CCP. For a given CCP, we denote the state of the process by $(k, x)$, if we have just observed the $k$ th object to be a candidate having value $x$, i.e. $X_{k}=L_{k}=x, 1 \leq k \leq n, 0<x<1$. The problem is specified by the functions $p_{k}(x)$ defined as the payoff earned by stopping in state $(k, x)$. For the best-choice problem, we 
can give $p_{k}(x)=x^{n-k}$ (payoff is now the probability of choosing the best), because, for the current candidate to be the best among all, each of the remaining observations $X_{k+1}, \ldots, X_{n}$ must be less than $x$. The version of the best-choice problem considered in Tamaki (2010, Section 4) is also a CCP with complicated $p_{k}(x)$; see Example 2.4 in Section 2. For the CCP, we use the same notation as used in the best-choice problem so long as no confusion occurs. For example, $v_{n}(\boldsymbol{a})$ represents the expected payoff under a monotone rule $\tau_{n}(\boldsymbol{a})$. One of the aims of this paper is to give two simple formulae for calculating $v_{n}(a)$ of the CCP; one is a formula related to the stopping time $\tau_{n}$ (formula of type 1) and the other a formula related to the time at which the largest value observed so far initially exceeds the threshold (formula of type 2). Ferguson et al. (1992, Sections 3.1 and 3.2) showed that both the duration problem and the best-choice duration problem are CCPs. The duration problem is concerned with maximizing the expected duration of holding a candidate. That is, if we stop with a candidate, we receive a payoff of 1 plus the number of future observations before a new candidate appears or until the final stage $n$ is reached. The best-choice duration problem is concerned with maximizing the expected duration of holding the best object (i.e. the last candidate). Hence, we have $p_{k}(x)=\sum_{j=0}^{n-k} x^{j} / n$ for the former and $p_{k}(x)=(n-k+1) x^{n-k} / n$ for the latter (note that, as is usual with them, the payoffs are divided by $n$ for normalization). Ferguson et al. (1992) mainly studied the optimal stopping rules for these two problems, but gave neither their expected payoffs nor their asymptotics.

As for the duration problem, the detailed analysis was made by Samuels (2004), Gnedin (2004), and Mazalov and Tamaki (2006) (see also Kurushima and Ano (2009) and Pearce et al. (2012)). However, the corresponding results for the best-choice duration problem have yet to be obtained. This is our motivation for studying this problem in Section 3. We derive the optimal expected payoff and show that it converges to the limiting value of 0.310965 as $n$ tends to $\infty$. Interestingly, this value can be obtained directly from the existing result of SamuelCahn (1996) if we recognize the equivalence between the best-choice duration problem and the Samuel-Cahn best-choice problem with uniform freeze. The same result can be also obtained via the planar Poisson process (PPP) model developed by Gnedin (1996), (2004), and Samuels (2004), which is known to be an appropriate setting in which we can define the infinite version of the corresponding finite problems (see also Bruss and Rogers (1991)). When recall is allowed, in which case any of the previously observed objects may be selected, the limiting payoff is shown to increase up to 0.335360 .

\section{Calculating the expected payoff of the CCP}

\subsection{Formula related to $\tau_{n}$}

We start with deriving the distribution of the stopping time $\tau_{n}(a)$ defined in (1.1).

Lemma 2.1. Assume that $n \geq 2$ and define, for a given monotone sequence of thresholds $\boldsymbol{a}=\left(a_{1}, a_{2}, \ldots, a_{n}\right)$,

$$
A_{k}(\boldsymbol{a})=\frac{1}{k} \sum_{i=1}^{k} a_{i}^{k}, \quad 1 \leq k<n
$$

with $A_{0}(\boldsymbol{a}) \equiv 1$ and $A_{n}(\boldsymbol{a}) \equiv 0$ for convention. We have the following:

(i) $\mathbb{P}\left\{\tau_{n}(\boldsymbol{a})>k\right\}=A_{k}(\boldsymbol{a}), 0 \leq k \leq n$;

(ii) $\mathbb{P}\left\{\tau_{n}(\boldsymbol{a})=k\right\}=A_{k-1}(\boldsymbol{a})-A_{k}(\boldsymbol{a}), 1 \leq k \leq n$;

(iii) $\mathbb{E}\left[\tau_{n}(\right.$ a $\left.)\right]=\sum_{k=0}^{n-1} A_{k}($ a $)$. 
Proof of Lemma 2.1(i). An important observation is that, since $a_{j}$ is nonincreasing in $j$ by definition while $L_{j}$ is nondecreasing in $j$ by nature, $\tau_{n}(a)>k$ occurs if and only if the largest of the first $k$ observations is less than the corresponding threshold. More specifically, if we let $T$ be the time index for which $L_{k}=X_{T}$, then

$$
\mathbb{P}\left\{\tau_{n}(\boldsymbol{a})>k\right\}=\mathbb{P}\left\{X_{T}<a_{T}\right\}
$$

Since $T$ is uniform on $\{1,2, \ldots, k\}$, by conditioning on $T$, we have

$$
\mathbb{P}\left\{X_{T}<a_{T}\right\}=\sum_{i=1}^{k} \mathbb{P}\left\{X_{T}<a_{T} \mid T=i\right\} \mathbb{P}\{T=i\}=\sum_{i=1}^{k}\left(a_{i}\right)^{k} \frac{1}{k},
$$

which proves Lemma 2.1(i) for $1 \leq k<n$. The results for $k=0$ and $n$ are evident. Lemmas 2.1(ii) and 2.1(iii) are immediate from Lemma 2.1(i), because $\mathbb{P}\left\{\tau_{n}(\boldsymbol{a})=k\right\}=$ $\mathbb{P}\left\{\tau_{n}(\boldsymbol{a})>k-1\right\}-\mathbb{P}\left\{\tau_{n}(\boldsymbol{a})>k\right\}$ and $\mathbb{E}\left[\tau_{n}(\boldsymbol{a})\right]=\sum_{k=0}^{n-1} \mathbb{P}\left\{\tau_{n}(\boldsymbol{a})>k\right\}$. Thus the proof is complete.

From Lemma 2.1(iii) we obtain (1.5), because (1.4) can be expressed as

$$
v_{n}^{*}=\left(\frac{1}{n}\right) \sum_{k=0}^{n-1} A_{k}\left(a^{*}\right) .
$$

This coincidence was pointed out in Mazalov and Tamaki (2006, Remark 3.3) in the limiting case.

We turn to the derivation of a formula for calculating $v_{n}(\boldsymbol{a})$ of the CCP. Before doing so, remember the well known result that, if $Z_{1}, Z_{2}, \ldots, Z_{m}$ are $m$ independent random variables each uniformly distributed on $(0, t)$ for a given $t>0$, then $\max \left\{Z_{1}, Z_{2}, \ldots, Z_{m}\right\}$ has a density given by

$$
g^{(m)}(z \mid t)=\frac{m}{t}\left(\frac{z}{t}\right)^{m-1}, \quad 0<z<t .
$$

Theorem 2.1. (Formula of type 1.) Let $p_{k}(x)$ be the payoff earned by stopping in state $(k, x)$. Then the expected payoff $v_{n}(\boldsymbol{a})$ under the monotone rule $\tau_{n}=\tau_{n}(\boldsymbol{a})$ is calculated from, for $n \geq 2$,

$$
v_{n}(\boldsymbol{a})=\int_{a_{1}}^{1} p_{1}(x) \mathrm{d} x+\sum_{k=2}^{n} \sum_{j=1}^{k-1} \int_{a_{k}}^{1} p_{k}(x) \frac{\left[\min \left(x, a_{j}\right)\right]^{k-1}}{k-1} \mathrm{~d} x .
$$
Then

Proof. Let $p(k, x)=p\left(\tau_{n}=k, L_{\tau_{n}}=x\right)$ be the joint probability density of $\left(\tau_{n}, L_{\tau_{n}}\right)$.

$$
v_{n}(\boldsymbol{a})=\sum_{k=1}^{n} \int_{a_{k}}^{1} p_{k}(x) p(k, x) \mathrm{d} x
$$

Obviously, $p(1, x) \equiv 1$. So, to prove (2.1), it suffices to show that, for $k \geq 2$,

$$
p(k, x)=\left[\sum_{j=1}^{k-1} \frac{1}{k-1} \int_{0}^{\min \left(x, a_{j}\right)} g^{(k-1)}(y \mid x) \mathrm{d} y\right] \frac{1}{k} g^{(k)}(x \mid 1) .
$$

Considering that the event $\left(\tau_{n}=k, L_{\tau_{n}}=x\right)$ can be equivalently described as $\left(X_{T}<a_{T}, L_{k}=\right.$ $X_{k}=x$ ), where $T$ is the time index for which $L_{k-1}=X_{T}$, because, to be $\tau_{n}=k$, the largest 
of the first $k-1$ observations must be less than the corresponding threshold (see the proof of Lemma 2.1), we can write

$$
\begin{aligned}
p\left(\tau_{n}=k, L_{\tau_{n}}=x\right) & =p\left(X_{T}<a_{T}, L_{k}=X_{k}=x\right) \\
& =\mathbb{P}\left\{X_{T}<a_{T} \mid L_{k}=X_{k}=x\right\} p\left(L_{k}=X_{k}=x\right) .
\end{aligned}
$$

We easily see that

$$
p\left(L_{k}=X_{k}=x\right)=\frac{1}{k} g^{(k)}(x \mid 1),
$$

because $g^{(k)}(x \mid 1)$ is the density of $L_{k}$ and $1 / k$ is the probability that the $k$ th observation is just the largest of the first $k$ observations. On the other hand, by conditioning on $T$, we have

$$
\begin{aligned}
\mathbb{P}\left\{X_{T}\right. & \left.<a_{T} \mid L_{k}=X_{k}=x\right\} \\
& =\sum_{j=1}^{k-1} \mathbb{P}\left\{X_{T}<a_{T} \mid T=j, L_{k}=X_{k}=x\right\} \mathbb{P}\left\{T=j \mid L_{k}=X_{k}=x\right\} \\
& =\sum_{j=1}^{k-1}\left[\int_{0}^{\min \left(x, a_{j}\right)} g^{(k-1)}(y \mid x) \mathrm{d} y\right]\left(\frac{1}{k-1}\right),
\end{aligned}
$$

where the last equality follows because $T$ is uniform on $\{1,2, \ldots, k-1\}$ independent of $L_{k}=X_{k}=x$, and because, given $L_{k}=X_{k}=x$ and $T=j, L_{k-1}\left(=X_{T}\right)$ can be viewed as the maximum of $k-1$ independent random variables each uniformly distributed on $(0, x)$ and the corresponding threshold is $a_{j}$. Applying (2.4) and (2.5) to (2.3) yields (2.2). This completes the proof.

Suppose that we are in state $(k, x)$ of a CCP with $p_{k}(x), 1 \leq k \leq n$. If we stop with the current candidate, we receive $p_{k}(x)$, while if we leave this state and stop with the next candidate, if any, we can expect to receive the payoff

$$
q_{k}(x)=\sum_{j=k+1}^{n} x^{j-k-1} \int_{x}^{1} p_{j}(y) \mathrm{d} y,
$$

where the vacuous sum is assumed to be 0 , i.e. $q_{n}(x)=0$.

Now let

$$
G=\bigcup_{k=1}^{n}\left\{(k, x): p_{k}(x) \geq q_{k}(x)\right\} .
$$

Hence, $G$ represents the set of states for which stopping immediately is at least as good as waiting for the next candidate to appear and then stopping. The stopping rule which stops as soon as the state enters the set $G$ is called one-stage look-ahead rule (1-sla rule). It is well known (see, e.g. Ferguson (2006) or Chow et al. (1971)) that the stopping rule $\tau_{n}\left(a^{*}\right)$, which is also 1-sla, is optimal if there exists a monotone sequence $\boldsymbol{a}^{*}=\left(a_{1}^{*}, a_{2}^{*}, \ldots, a_{n}^{*}\right)$ such that $G$ can be expressed as $G=\bigcup_{k=1}^{n}\left\{(k, x): x \geq a_{k}^{*}\right\}$. Thus, we refer to the stopping problem as a $\mathrm{CCP}$ when it has an optimal 1-sla rule.

Let

$$
r=\min \left\{k: p_{k}(x) \geq q_{k}(x), 0 \leq x \leq 1\right\}
$$


When the $p_{k}(x) \mathrm{s}$ are continuous in $x, a_{k}^{*}$ is clearly given as a root $x$ of $p_{k}(x)=q_{k}(x)$, i.e.

$$
p_{k}(x)=\sum_{j=k+1}^{n} x^{j-k-1} \int_{x}^{1} p_{j}(y) \mathrm{d} y
$$

for $k<r$ and $a_{k}^{*}=0$ for $r \leq k \leq n$. Once we have obtained $a_{k}^{*}$ inductively as a unique root $x \in\left(a_{k+1}^{*}, 1\right)$ of $(2.7)$, the optimal (expected) payoff is given as $v_{n}^{*}=v_{n}\left(a^{*}\right)$ by substituting $\boldsymbol{a}^{*}$ for $\boldsymbol{a}$ in (2.1). We give some examples and comments.

Example 2.1. (Best-choice problem: $p_{k}(x)=x^{n-k}$ and $r=n$.) Straightforward calculations from (2.1) yields

$$
v_{n}(\boldsymbol{a})=\frac{1}{n}\left[1+\sum_{k=1}^{n-1} \sum_{j=k}^{n-1} \frac{1}{j} a_{k}^{j}+\sum_{k=1}^{n-1}\left\{\sum_{j=k}^{n-1} \frac{1}{n-j} a_{k}^{j}-\left(1+h_{n-k}\right) a_{k}^{n}\right\}-a_{n}^{n}\right],
$$

where $h_{k}=\sum_{j=1}^{k} 1 / j, k \geq 1$ with $h_{0}=0$. It is easy to check that this agrees with (1.2). From (1.3), $a_{k}^{*}$ satisfies

$$
\sum_{j=k}^{n-1} \frac{1}{n-j}\left(a_{k}^{*}\right)^{j}=\left(1+h_{n-k}\right)\left(a_{k}^{*}\right)^{n}, \quad 1 \leq k<r .
$$

Applying these to the above, combined with $a_{n}^{*}=0$, immediately yields (1.4).

Example 2.2. (Duration problem: $p_{k}(x)=\sum_{j=0}^{n-k} x^{j} / n$ and $r=n-1$.) We obtain from (2.1), after tedious calculations,

$$
v_{n}(\boldsymbol{a})=\frac{1}{n}\left[h_{n}+\sum_{k=1}^{n} \sum_{j=k}^{n} \frac{1}{j}\left(h_{n-j}-h_{j-k}-1\right) a_{k}^{j}\right] .
$$

Mazalov and Tamaki (2006) obtained this expression, not from (2.1), but from an alternative formula (2.8) given below in Theorem 2.2. From (2.7), $a_{k}^{*}$ satisfies

$$
\sum_{j=k}^{n}\left(h_{n-j}-h_{j-k}-1\right)\left(a_{k}^{*}\right)^{j}=0, \quad 1 \leq k<r .
$$

Example 2.3. (Best-choice duration problem: $p_{k}(x)=(n-k+1) x^{n-k} / n$ and $r=n$.) See Lemma 3.1 for $v_{n}(\boldsymbol{a})$ and $\left\{a_{k}^{*}\right\}$.

Example 2.4. (Version of the best-choice problem.) The problem considered in Tamaki (2010, Section 4) is concerned with maximizing the probability of stopping with any of the last $m$ candidates, where $m$ is a predetermined positive integer. When $m=1$, this problem reduces to the best-choice problem. Let $r_{j}(k), 1 \leq k \leq j$, be defined recursively as

$$
r_{j}(k)=\frac{1}{j} r_{j-1}(k-1)+\left(1-\frac{1}{j}\right) r_{j-1}(k), \quad 1 \leq k \leq j, 2 \leq j
$$

with $r_{1}(1)=1$ and $r_{j}(k)=0$ for $k=0$ or $k>j$, and define $d_{j}=\sum_{k=1}^{m-1} r_{j}(k)$ for $j \geq m$ and $d_{j}=1$ for $j<m$. Then we have, for $k<r=n+1-m$,

$$
p_{k}(x)=\sum_{j=0}^{n-k} d_{j}\left(\begin{array}{c}
n-k \\
j
\end{array}\right)(1-x)^{j} x^{n-k-j} .
$$


The expected payoff $v_{n}(\boldsymbol{a})$ is given as $P_{n, m}^{*}$ in Tamaki (2010, Theorem 4.1(b)) if $b_{j}(m)$ is replaced by $a_{n-j}$ for each $j$.

\subsection{Formula related to $\sigma_{n}$}

Besides (2.1), we can obtain another expression for $v_{n}(a)$. Let $\sigma_{n}(a)$ be the time at which the largest value observed so far initially exceeds the threshold, i.e.

$$
\sigma_{n}=\sigma_{n}(\boldsymbol{a})=\min \left\{k: L_{k} \geq a_{k}\right\} \wedge n .
$$

By definition, $\mathbb{P}\left\{\sigma_{n}(\boldsymbol{a}) \leq \tau_{n}(\boldsymbol{a})\right\}=1$. It is easy to see that $\sigma_{n}(\boldsymbol{a})>k$ is equivalent to $L_{k}<a_{k}$ due to the monotonicity of $a$. Hence, we immediately have the following results concerning the distribution of $\sigma_{n}(\boldsymbol{a})$.

Lemma 2.2. Assume that $n \geq 2$. Then, for a given monotone sequence $\boldsymbol{a}=\left(a_{1}, a_{2}, \ldots, a_{n}\right)$, we have the following:

(i) $\mathbb{P}\left\{\sigma_{n}(a)>k\right\}=a_{k}^{k}, 0 \leq k \leq n$;

(ii) $\mathbb{P}\left\{\sigma_{n}(\boldsymbol{a})=k\right\}=a_{k-1}^{k-1}-a_{k}^{k}, 1 \leq k \leq n$;

(iii) $\mathbb{E}\left[\sigma_{n}(\boldsymbol{a})\right]=\sum_{k=0}^{n-1} a_{k}^{k}$,

where $a_{0}=1$ and $a_{n}^{n}=0$ for convention.

Another expression for $v_{n}(\boldsymbol{a})$ is given as follows.

Theorem 2.2. (Formula of type 2.) Let $q_{k}(x)$ be as defined in (2.6). Then, we have

$$
v_{n}(\boldsymbol{a})=\sum_{k=1}^{n}\left[\int_{a_{k}}^{1} p_{k}(x)\left[\min \left(x, a_{k-1}\right)\right]^{k-1} \mathrm{~d} x+(k-1) \int_{a_{k}}^{a_{k-1}} q_{k}(x) x^{k-1} \mathrm{~d} x\right] .
$$

Proof. Let $p(k, x)=p\left(\sigma_{n}=k, L_{\sigma_{n}}=x\right)$ be the joint probability density of $\left(\sigma_{n}, L_{\sigma_{n}}\right)$. To obtain $p(k, x)$, we distinguish two cases according to whether $L_{\sigma_{n}}=X_{\sigma_{n}}$ (case 1), or $L_{\sigma_{n}}>X_{\sigma_{n}}$ (case 2 ), and denote $p(k, x)$ by $p_{1}(k, x)$ for case 1 and by $p_{2}(k, x)$ for case 2 . Then we obtain, for $k \geq 2$,

$$
\begin{aligned}
p_{1}(k, x)= & {\left[\int_{0}^{\min \left(x, a_{k-1}\right)} g^{(k-1)}(y \mid x) \mathrm{d} y\right] \frac{1}{k} g^{(k)}(x \mid 1), \quad a_{k} \leq x \leq 1, } \\
& p_{2}(k, x)=\frac{k-1}{k} g^{(k)}(x \mid 1), \quad a_{k} \leq x<a_{k-1},
\end{aligned}
$$

in a similar way to that used to derive (2.2) in the proof of Theorem 2.1. For $k=1$, clearly $p_{1}(k, x) \equiv 1$ and $p_{2}(k, x) \equiv 0$. Note that the inside of the bracket of $p_{1}(k, x)$ is the probability that the largest of the first $k-1$ observations must be less than $a_{k-1}$ conditional on $L_{k}=X_{k}=x$. On the event ( $\sigma_{n}=k, L_{\sigma_{n}}=x$ ), we stop with the current candidate and receive the payoff $p_{k}(x)$ for case 1 , whereas we continue observations and stop with the next candidate, if any, and receive the payoff $q_{k}(x)$ for case 2 . Hence, we have

$$
v_{n}(\boldsymbol{a})=\sum_{k=1}^{n}\left[\int_{a_{k}}^{1} p_{k}(x) p_{1}(k, x) \mathrm{d} x+\int_{a_{k}}^{a_{k-1}} q_{k}(x) p_{2}(k, x) \mathrm{d} x\right],
$$

which yields (2.8), completing the proof.

Remark 2.1. Formula of type 2 is applicable to some problems with recall. See Section 3.2 and Mazalov and Tamaki (2006, Section 2.2). 


\subsection{Limiting expected values of $\tau_{n} / n$ and $\sigma_{n} / n$}

To make explicit the dependence on $n$, we sometimes write $\boldsymbol{a}(n)=\left(a_{1}(n), a_{2}(n), \ldots, a_{n}(n)\right)$ instead of $\boldsymbol{a}=\left(a_{1}, a_{2}, \ldots, a_{n}\right)$. Let $\left\{b_{k}\right\}_{k=0}^{\infty}$ be an infinite sequence such that

$$
0 \leq b_{0} \leq b_{1} \leq b_{2} \leq \cdots \leq 1, \quad \lim _{n \rightarrow \infty} n\left(1-b_{n}\right)=c<\infty
$$

and define $a_{k}(n)=b_{n-k}, 1 \leq k \leq n$, i.e. $\boldsymbol{a}(n)=\left(b_{n-1}, b_{n-2}, \ldots, b_{0}\right), n \geq 1$. Then we have the following limiting results.

Lemma 2.3. For $\boldsymbol{a}(n)$ satisfying (2.9),

(i) it holds that

$$
\lim _{n \rightarrow \infty} \mathbb{E}\left[\frac{\tau_{n}(\boldsymbol{a}(n))}{n}\right]=\mathrm{e}^{-c}+\left(\mathrm{e}^{c}-c-1\right) I(c)
$$

(ii) and

$$
\lim _{n \rightarrow \infty} \mathbb{E}\left[\frac{\sigma_{n}(\boldsymbol{a}(n))}{n}\right]=1-c \mathrm{e}^{c} I(c) .
$$

Proof of Lemma 2.3(i). Let $c_{j}=j\left(1-b_{j}\right), j \geq 0$. Then, from Lemma 2.1(iii), we have

$$
\begin{aligned}
\mathbb{E}\left[\frac{\tau_{n}(a(n))}{n}\right] & =\frac{1}{n}\left[1+\sum_{k=1}^{n-1} \sum_{i=1}^{k} \frac{1}{k}\left(b_{n-i}\right)^{k}\right] \\
& =\frac{1}{n}\left[1+\sum_{j=1}^{n-1} \sum_{r=1}^{j} \frac{1}{n-r}\left(b_{j}\right)^{n-r}\right] \\
& =\frac{1}{n}\left[1+\sum_{j=1}^{n-1} \sum_{r=1}^{j} \frac{1}{n-r}\left(1-\frac{c_{j}}{j}\right)^{n-r}\right] .
\end{aligned}
$$

Since $c_{j} \rightarrow c$ as $j \rightarrow \infty$, it is easy to see that, as $n \rightarrow \infty$,

$$
\mathbb{E}\left[\frac{\tau_{n}(\boldsymbol{a}(n))}{n}\right] \rightarrow \int_{0}^{1} \int_{0}^{u} \frac{1}{1-v}\left(\mathrm{e}^{-c}\right)^{(1-v) / u} \mathrm{~d} v \mathrm{~d} u
$$

For the transformation from the bivariate integral of (2.12) to the right-hand side of (2.10), see Tamaki (2009, Appendix).

Proof of Lemma 2.3(ii). From Lemma 2.2(iii), we similarly have

$$
\mathbb{E}\left[\frac{\sigma_{n}(\boldsymbol{a}(n))}{n}\right]=\frac{1}{n}\left[1+\sum_{j=1}^{n-1}\left(1-\frac{c_{j}}{j}\right)^{n-j}\right] \rightarrow \int_{0}^{1}\left(\mathrm{e}^{-c}\right)^{(1-u) / u} \mathrm{~d} u=\mathrm{e}^{c} \int_{0}^{1} \mathrm{e}^{-c / u} \mathrm{~d} u
$$

Let $v=1 / u$. Then

$$
\int_{0}^{1} \mathrm{e}^{-c / u} \mathrm{~d} u=\int_{1}^{\infty} \frac{\mathrm{e}^{-c v}}{v^{2}} \mathrm{~d} v=\mathrm{e}^{-c}-c I(c),
$$

where the second equality follows from integration by parts. Substituting (2.14) into (2.13) gives (2.11). 
Remark 2.2. It is noted that the bivariate integral of (2.12) with $c \approx 1.3450$ appeared in Mazalov and Tamaki (2006, Equation (1.2)) as the limiting optimal payoff of the duration problem with recall.

The threshold sequence $a(n)$ considered in Lemma 2.3 assumes that the threshold value $a_{k}(n)$ depends on $k$ only through the number of remaining observations $n-k$. This is the case with the optimal threshold sequence of the problems given in the following examples. The corresponding limiting value $c$ is listed below for reference.

(i) Best-choice problem: $c \approx 0.80435$ is a solution $t$ to the equation $J(t)=1$; see Gilbert and Mosteller (1966).

(ii) Duration problem: $c \approx 2.1198$ is a solution $t$ to the equation $\mathrm{e}^{t}[1+J(-t)]=1-J(t)$; see Ferguson et al. (1992).

(iii) Best-choice duration problem: $c \approx 1.25643$ is a solution $t$ to the equation $\mathrm{e}^{t}=1+2 t$; see Ferguson et al. (1992).

(iv) Version of the best-choice problem: $c$ is a solution $t$ to the equation $\sum_{i=m}^{\infty} r_{i}(m) t^{i} / i$ ! $=1$ for a given $m$; see Tamaki (2010).

\section{Best-choice duration problem}

The study on the best-choice duration problem in Ferguson et al. (1992, Section 3.2) was quite preliminary. Here we reconsider this problem in detail both in the case where the most recently observed object may be selected (i.e. the no recall case) and in the case where any of the previously observed objects may be selected (i.e. recall case).

\subsection{No recall case}

This problem is a CCP and the next lemma yields the expected payoff.

Lemma 3.1. (Expected payoff in the no recall case.) (i) We have

$$
v_{n}(\boldsymbol{a})=\frac{1}{n}\left[1+\sum_{k=1}^{n-1} \sum_{j=k}^{n-1} \frac{a_{k}^{j}}{j}-\frac{1}{n} \sum_{k=1}^{n}(2(n-k)+1) a_{k}^{n}\right]
$$

(ii) and

$$
v_{n}^{*}=\frac{1}{n}\left[1+\sum_{k=1}^{n-1} \sum_{j=k}^{n-1}\left(\frac{1}{j}-\frac{1}{n}\right)\left(a_{k}^{*}\right)^{j}\right],
$$

where $a_{n}^{*}=0$ (i.e. $r=n$ ) and $a_{k}^{*}, k<n$, is a unique solution $x \in(0,1)$ of

$$
(2(n-k)+1) x^{n}=\sum_{i=k}^{n-1} x^{i}
$$

Proof. We have $p_{k}(x)=(n-k+1) x^{n-k} / n$, because, when we stop in state $(k, x)$, the payoff is $(n-k+1) / n$ or 0 depending on whether or not the current candidate is best overall. Hence, (3.3) is obtained from (2.7) (for these, see also Ferguson et al. (1992), where time is taken as the remaining time to the end). 
We now prove Lemma 3.1(i). Since

$$
\begin{aligned}
\int_{a_{k}}^{1} p_{k}(x) \frac{\left[\min \left(x, a_{j}\right)\right]^{k-1}}{k-1} \mathrm{~d} x & =\frac{n-k+1}{n(k-1)}\left(\int_{a_{k}}^{a_{j}} x^{n-1} \mathrm{~d} x+a_{j}^{k-1} \int_{a_{j}}^{1} x^{n-k} \mathrm{~d} x\right) \\
& =\frac{1}{n}\left[\frac{1}{k-1} a_{j}^{k-1}-\frac{1}{n} a_{j}^{n}-\frac{n-k+1}{n(k-1)} a_{k}^{n}\right],
\end{aligned}
$$

from (2.1), we have

$$
\begin{aligned}
v_{n}(\boldsymbol{a}) & =\frac{1}{n}\left[\int_{a_{1}}^{1} n x^{n-1} \mathrm{~d} x+\sum_{k=2}^{n} \sum_{j=1}^{k-1}\left(\frac{1}{k-1} a_{j}^{k-1}-\frac{1}{n} a_{j}^{n}-\frac{n-k+1}{n(k-1)} a_{k}^{n}\right)\right] \\
& =\frac{1}{n}\left[\left(1-a_{1}^{n}\right)+\sum_{k=1}^{n-1} \sum_{j=1}^{k} \frac{1}{k} a_{j}^{k}-\frac{1}{n} \sum_{j=1}^{n}(n-j) a_{j}^{n}-\frac{1}{n} \sum_{k=2}^{n}(n-k+1) a_{k}^{n}\right],
\end{aligned}
$$

which gives (3.1) after some arrangement.

We now prove Lemma 3.1(ii). We obtain (3.2) by applying to the second term on the right-hand side of (3.1),

$$
\sum_{k=1}^{n-1}(2(n-k)+1)\left(a_{k}^{*}\right)^{n}=\sum_{k=1}^{n-1} \sum_{i=k}^{n-1}\left(a_{k}^{*}\right)^{i},
$$

which follows from (3.3).

Moreover, the relation between the optimal payoff $v_{n}^{*}$ and the first two moments of the optimal proportional stopping time $\tau_{n}^{*} / n$ is given as follows.

Lemma 3.2. We have

$$
v_{n}^{*}=\left(1+\frac{1}{2 n}\right) \mathbb{E}\left[\frac{\tau_{n}^{*}}{n}\right]-\frac{1}{2} \mathbb{E}\left[\left(\frac{\tau_{n}^{*}}{n}\right)^{2}\right]
$$

Proof. From (3.2),

$$
v_{n}^{*}=\frac{1}{n}\left[1+\sum_{k=1}^{n-1} \sum_{j=k}^{n-1} \frac{1}{j}\left(a_{k}^{*}\right)^{j}\right]-\frac{1}{n^{2}} \sum_{k=1}^{n-1} \sum_{j=k}^{n-1}\left(a_{k}^{*}\right)^{j} .
$$

The first term on the right-hand side of (3.5) is $\mathbb{E}\left[\tau_{n}^{*} / n\right]$ as seen in (1.5). The double summation of the second term can be written as, from Lemma 2.1(i),

$$
\begin{aligned}
\sum_{k=1}^{n-1} \sum_{j=k}^{n-1}\left(a_{k}^{*}\right)^{j} & =\sum_{j=1}^{n-1}\left(\sum_{k=1}^{j}\left(a_{k}^{*}\right)^{j}\right) \\
& =\sum_{j=1}^{n-1} j A_{j}\left(\boldsymbol{a}^{*}\right) \\
& =\sum_{j=1}^{n-1} j \mathbb{P}\left\{\tau_{n}^{*}>j\right\} \\
& =\frac{1}{2}\left(\mathbb{E}\left[\left(\tau_{n}^{*}\right)^{2}\right]-\mathbb{E}\left[\tau_{n}^{*}\right]\right),
\end{aligned}
$$


where the last equality follows from the well known fact that, for a nonnegative integer-valued random variable $X$,

$$
\sum_{j=0}^{\infty} j \mathbb{P}\{X>j\}=\frac{1}{2}\left(\mathbb{E}\left[X^{2}\right]-\mathbb{E}[X]\right) .
$$

Applying the above results to (3.5) yields (3.4).

Samuel-Cahn (1996) generalized the best-choice problem by introducing a random 'freezetime' variable $N$ which makes it impossible to make a selection after time $N$. The goal of stopping with the largest of $X_{1}, X_{2}, \ldots, X_{n}$ remains unchanged. In the case of $N$ uniform on $\{1,2, \ldots, n\}$, Samuel-Cahn showed that the optimal rule is a monotone rule with $a^{*}=$ $\left(a_{1}^{*}, a_{2}^{*}, \ldots, a_{n}^{*}\right)$, where $a_{k}^{*}$ is determined from (3.3) and the optimal probability is given by $v_{n}\left(a^{*}\right)$, where $v_{n}(a)$ is given by (3.1). That is, the best-choice duration problem (without recall) is equivalent to the Samuel-Cahn best-choice problem with $N$ uniform on $\{1,2, \ldots, n\}$. From this equivalence, we can obtain an integral expression for $v^{*}$.

Lemma 3.3. (Limiting optimal payoff.) We have

$$
v^{*}=\int_{0}^{1} \frac{1}{x}\left[\int_{0}^{x} \mathrm{e}^{-c^{*} x /(1-y)} \mathrm{d} y\right] \mathrm{d} x-2 \int_{0}^{1} y \mathrm{e}^{-c^{*} / y} \mathrm{~d} y \approx 0.310965,
$$

where $c^{*}(\approx 1.25643)$ is a unique solution $c(>0)$ of

$$
\mathrm{e}^{c}=1+2 c
$$

Proof. See Samuel-Cahn (1996, Section 4), where $v^{*}$ of (3.6) was derived in an ingenious way, starting from the form of (3.1), combined with (3.3). See also Ferguson et al. (1992) for (3.7).

Another simpler expression for $v^{*}$ in terms of $I(c)$ is given as follows.

Lemma 3.4. Write $c$ for $c^{*}$ for convenience. Then

$$
v^{*}=c \mathrm{e}^{-c}+c(1-c) I(c) .
$$

Proof. Let $v=x /(1-y)$. Then

$$
\int_{0}^{x} \mathrm{e}^{-c x /(1-y)} \mathrm{d} y=x \int_{x}^{x /(1-x)} \frac{\mathrm{e}^{-c v}}{v^{2}} \mathrm{~d} v .
$$

Interchanging the order of integration, the double integral of (3.6) becomes

$$
\begin{aligned}
\int_{0}^{1}\left[\int_{x}^{x /(1-x)} \frac{\mathrm{e}^{-c v}}{v^{2}} \mathrm{~d} v\right] \mathrm{d} x & =\int_{0}^{1}\left[\int_{v /(1+v)}^{v} \mathrm{~d} x\right] \frac{\mathrm{e}^{-c v}}{v^{2}} \mathrm{~d} v+\int_{1}^{\infty}\left[\int_{v /(1+v)}^{1} \mathrm{~d} x\right] \frac{\mathrm{e}^{-c v}}{v^{2}} \mathrm{~d} v \\
& =\int_{0}^{1} \frac{v^{2}}{1+v} \frac{\mathrm{e}^{-c v}}{v^{2}} \mathrm{~d} v+\int_{1}^{\infty} \frac{1}{1+v} \frac{\mathrm{e}^{-c v}}{v^{2}} \mathrm{~d} v \\
& =\int_{0}^{\infty} \frac{\mathrm{e}^{-c v}}{1+v} \mathrm{~d} v-\int_{1}^{\infty} \frac{\mathrm{e}^{-c v}}{v} \mathrm{~d} v+\int_{1}^{\infty} \frac{\mathrm{e}^{-c v}}{v^{2}} \mathrm{~d} v \\
& =\mathrm{e}^{c} I(c)-I(c)+\int_{1}^{\infty} \frac{\mathrm{e}^{-c v}}{v^{2}} \mathrm{~d} v .
\end{aligned}
$$


As seen in (2.14), integration by parts gives

$$
\int_{1}^{\infty} \frac{\mathrm{e}^{-c v}}{v^{2}} \mathrm{~d} v=\mathrm{e}^{-c}-c I(c) .
$$

Let $1 / y=v$. Then the integral of the second term of (3.6) is written as, again by integration by parts,

$$
\int_{1}^{\infty} \frac{\mathrm{e}^{-c v}}{v^{3}} \mathrm{~d} v=\frac{\mathrm{e}^{-c}}{2}-\frac{c}{2} \int_{1}^{\infty} \frac{\mathrm{e}^{-c v}}{v^{2}} \mathrm{~d} v
$$

Applying (3.9)-(3.11) to (3.6) yields (3.8).

The asymptotic result (3.8) is also obtained via the PPP model. According to Samuels (2004), we use a Poisson process with unit rate on the semi-infinite strip $[0,1] \times[0, \infty)$. This turns the problem upside down, turning the 'best' into the 'smallest'. The process is scanned from left to right by shifting a vertical detector and the scanning can be stopped each time a point in the PPP, referred to as an atom henceforth, is detected. A link to the finite problem can be established in a similar manner as given to the best-choice problem by Gnedin (1996).

Suppose that an atom is identified as a point $(t, y)$ if the atom appears at time $t$ as a candidate (relatively best atom as in the finite problem) having value $y$ in the PPP. Let $P(t, y)$ denote the expected payoff if we choose this point, i.e. stop on the point $(t, y)$. Then

$$
P(t, y)=(1-t) \mathrm{e}^{-y(1-t)},
$$

because $\mathrm{e}^{-y(1-t)}$ is the probability that no atom appears in the box domain $[t, 1] \times[0, y]$ whose area is $y(1-t)$. If we do not choose this point, but choose the point related to the next candidate, if any, then, since its value is uniformly distributed on $(0, y)$, we can expect to receive a payoff

$$
Q(t, y)=\int_{0}^{1-t}\left(\int_{0}^{y} P(t+r, x) \frac{1}{y} \mathrm{~d} x\right) y \mathrm{e}^{-y r} \mathrm{~d} r .
$$

Substituting (3.12) into (3.13) yields

$$
Q(t, y)=\frac{1}{y}\left(1-\mathrm{e}^{-y(1-t)}\right)-(1-t) \mathrm{e}^{-y(1-t)} .
$$

Solving for the locus of point $(t, y)$ at which $P(t, y)=Q(t, y)$ yields $y(1-t)=c^{*}$, where $c^{*} \approx 1.25643$ is as defined from (3.7). Since $P(t, y) \geq Q(t, y)$ implies that $P\left(t^{\prime}, y^{\prime}\right) \geq$ $Q\left(t^{\prime}, y^{\prime}\right)$ for $t^{\prime}>t, y^{\prime}<y$, we are in the monotone case of optimal stopping (see Ferguson (2006) or Chow et al. (1971) for the monotone case) and can conclude that the optimal rule stops with the first candidate, if any, that lies below the threshold curve $y=c^{*} /(1-t)$. Henceforth, we again write $c$ instead of $c^{*}$ for simplicity. Let $T$ be the arrival time of the first (leftmost) atom that lies below the threshold curve $y=c /(1-t)$, and let $S$ be the time when the value of the best (lowest) atom above threshold is equal to the threshold. Then it is easy to see that (see, e.g. Samuels (2004, Section 10.2)) the limiting optimal payoff is calculated from

$$
\begin{aligned}
v^{*}= & \int_{0}^{1} \int_{0}^{t} P\left(s, \frac{c}{1-s}\right) f_{S}(s) f_{T}(t) \mathrm{d} s \mathrm{~d} t \\
& +\int_{0}^{1} \int_{0}^{s}\left(\frac{1-t}{c} \int_{0}^{c /(1-t)} P(t, y) \mathrm{d} y\right) f_{T}(t) f_{S}(s) \mathrm{d} t \mathrm{~d} s
\end{aligned}
$$


where $f_{T}(t)$ and $f_{S}(s)$ are the densities of $T$ and $S$ given as

$$
f_{T}(t)=c(1-t)^{c-1}, \quad f_{S}(s)=\frac{c s}{(1-s)^{c+2}} \mathrm{e}^{-c s /(1-s)} .
$$

Applying (3.12) to (3.14) yields

$$
v^{*}=\mathrm{e}^{-c} \int_{0}^{1} \int_{0}^{t}(1-s) f_{S}(s) f_{T}(t) \mathrm{d} s \mathrm{~d} t+\frac{1-\mathrm{e}^{-c}}{c} \int_{0}^{1} \int_{0}^{s}(1-t) f_{T}(t) f_{S}(s) \mathrm{d} t \mathrm{~d} s .
$$

Moreover, the bivariate integrals can be simplified to

$$
\begin{gathered}
\int_{0}^{1} \int_{0}^{t}(1-s) f_{S}(s) f_{T}(t) \mathrm{d} s \mathrm{~d} t=c\left[(1+c) \mathrm{e}^{c} I(c)-1\right], \\
\int_{0}^{1} \int_{0}^{s}(1-t) f_{T}(t) f_{S}(s) \mathrm{d} s \mathrm{~d} t=c\left[1-c \mathrm{e}^{c} I(c)\right]
\end{gathered}
$$

respectively. Substituting (3.16) and (3.17) into (3.15), we obtain

$$
v^{*}=1-(1+c) \mathrm{e}^{-c}+c\left(2+c-\mathrm{e}^{c}\right) I(c),
$$

and so (3.18), combined with (3.7), is readily reduced to (3.8). We thus have shown that the result (3.8) is obtained via the PPP approach.

Remark 3.1. The equivalence between the best-choice duration problem (without recall) and the Samuel-Cahn best-choice problem with uniform freeze is not surprising, because this is very similar to the equivalence between the duration problem (without recall) and the Porosinski (1987) best-choice problem with uniform horizon, to which Samuels (2004) and Gnedin (2004) have given a good explanation. See also Gnedin (2005) for further generalization of the equivalence.

\subsection{Recall case}

Ferguson et al. (1992) showed that, in the recall case, the optimal stopping rule is within the class of stopping rules $\left\{\sigma_{n}(\boldsymbol{a})\right\}$ in the sense that it stops at time $\sigma_{n}$ with the current candidate if $L_{\sigma_{n}}=X_{\sigma_{n}}$, but with the previous object, say, the $j$ th object if $L_{\sigma_{n}}=X_{j}>X_{\sigma_{n}}$. Moreover, they showed that the optimal threshold $a_{k}^{*}=2^{-1 /(n-k)}$ is given as a solution $x$ of $p_{k}(x)=x p_{k+1}(x)+\int_{x}^{1} p_{k+1}(y) \mathrm{d} y, k<n$, where $p_{k}(x)=(n-k+1) x^{n-k} / n$. Let $u_{n}(a)$ denote the expected payoff under $\sigma_{n}(\boldsymbol{a})$ and $u_{n}^{*}=u_{n}\left(\boldsymbol{a}^{*}\right)$. Then we have the following results.

Lemma 3.5. (Expected payoff in the recall case.) (i) We have

$$
u_{n}(\boldsymbol{a})=\frac{1}{n}\left[1+\sum_{k=1}^{n} a_{k}^{k}-2 \sum_{k=1}^{n} \frac{k}{n} a_{k}^{n}\right]
$$

(ii) and

$$
u_{n}^{*}=\frac{1}{n}\left[1+\sum_{k=1}^{n}\left(1-\frac{k}{n}\right) 2^{-k /(n-k)}\right]
$$


Proof of Lemma 3.5(i). A bit of consideration shows that $u_{n}(\boldsymbol{a})$ is obtained by simply replacing $q_{k}(x)$ by $p_{k}(x)$ in (2.8), because the stopping payoff at time $\sigma_{n}$ is always $p_{\sigma_{n}}\left(L_{\sigma_{n}}\right)$. That is,

$$
u_{n}(\boldsymbol{a})=\sum_{k=1}^{n}\left[\int_{a_{k}}^{1} p_{k}(x)\left[\min \left(x, a_{k-1}\right)\right]^{k-1} \mathrm{~d} x+(k-1) \int_{a_{k}}^{a_{k-1}} p_{k}(x) x^{k-1} \mathrm{~d} x\right] .
$$

From straightforward calculation, we obtain

$$
\begin{aligned}
\int_{a_{k}}^{1} p_{k}(x)\left[\min \left(x, a_{k-1}\right)\right]^{k-1} \mathrm{~d} x+(k-1) \int_{a_{k}}^{a_{k-1}} p_{k}(x) x^{k-1} \mathrm{~d} x \\
\quad=k \int_{a_{k}}^{a_{k-1}} p_{k}(x) x^{k-1} \mathrm{~d} x+a_{k-1}^{k-1} \int_{a_{k-1}}^{1} p_{k}(x) \mathrm{d} x \\
\quad=\frac{1}{n}\left[\frac{(k-1)(n-k)}{n} a_{k-1}^{n}-\frac{k(n-k-1)}{n} a_{k}^{n}-\frac{2 k}{n} a_{k}^{n}+a_{k-1}^{k-1}\right] .
\end{aligned}
$$

Substituting (3.21) into (3.20) yields (3.19). The proof of Lemma 3.5(ii) follows from $a_{k}^{*}=$ $2^{-1 /(n-k)}$.

Let $u^{*}=\lim _{n \rightarrow \infty} u_{n}^{*}$. Then we have the following limiting result.

Lemma 3.6. Let $\tilde{c}=\log 2$. Then

$$
u^{*}=\frac{1-\tilde{c}}{2}+(\tilde{c})^{2} I(\tilde{c}) \approx 0.335360 .
$$

Proof. From Lemma 3.5(ii), $u_{n}^{*}$ can be written as

$$
u_{n}^{*}=\frac{1}{n}\left[1+\sum_{k=1}^{n}\left(1-\frac{k}{n}\right)\left(\mathrm{e}^{-\tilde{c}}\right)^{k /(n-k)}\right]
$$

which yields, by letting $n \rightarrow \infty$,

$$
u^{*}=\int_{0}^{1}(1-x)\left(\mathrm{e}^{-\tilde{c}}\right)^{x /(1-x)} \mathrm{d} x .
$$

Let $v=1 /(1-x)$. Then

$$
\int_{0}^{1}(1-x)\left(\mathrm{e}^{-\tilde{c}}\right)^{x /(1-x)} \mathrm{d} x=\mathrm{e}^{\tilde{c}} \int_{1}^{\infty} \frac{1}{v^{3}} \mathrm{e}^{-\tilde{c} v} \mathrm{~d} v=\frac{1-\tilde{c}}{2}+\frac{(\tilde{c})^{2} \mathrm{e}^{\tilde{c}}}{2} I(\tilde{c}),
$$

where the last equality follows from (3.11) and (3.10). Hence, (3.23) reduces to (3.22) through $\mathrm{e}^{\tilde{c}}=2$.

Remark 3.2. We can also obtain $u^{*}$ of (3.22) from the PPP model, i.e. $u^{*}$ is just the value of $v^{*}$ of (3.15) when $c\left(=c^{*} \approx 1.25643\right)$ is replaced by $\tilde{c}=\log 2 \approx 0.69315$, because, as is easily seen from the argument of the infinitesimal look-ahead stopping rule used for the duration problem with recall in Mazalov and Tamaki (2006, Section 3.2), the optimal threshold curve in the recall case is given by $y=\tilde{c} /(1-t)$, in contrast to the curve $y=c^{*} /(1-t)$ in the no recall case $(\tilde{c}=\log 2$ was already suggested in Ferguson et al. (1992)). 
Let $\tau_{n}^{*}\left(\tilde{\sigma}_{n}\right)$ be the optimal stopping time of the best-choice duration problem with recall (without recall). Then, since the corresponding optimal thresholds $a_{k}^{*}(n)=b_{n-k}^{*}\left(\tilde{a}_{k}(n)=\tilde{b}_{n-k}\right)$ satisfy the property that $\lim _{n \rightarrow \infty} n\left(1-b_{n}^{*}\right)=c^{*}\left(\lim _{n \rightarrow \infty} n\left(1-\tilde{b}_{n}\right)=\tilde{c}\right)$, we immediately have the following results from Lemma 2.3.

Lemma 3.7. Let $c^{*} \approx 1.25643$ and $\tilde{c}=\log 2$. Then

$$
\begin{gathered}
\lim _{n \rightarrow \infty} \mathbb{E}\left[\frac{\tau_{n}^{*}}{n}\right]=\mathrm{e}^{-c^{*}}+\left(\mathrm{e}^{c^{*}}-c^{*}-1\right) I\left(c^{*}\right) \approx 0.46678, \\
\lim _{n \rightarrow \infty} \mathbb{E}\left[\frac{\tilde{\sigma}_{n}}{n}\right]=1-\tilde{c} \mathrm{e}^{\tilde{c}} I(\tilde{c}) \approx 0.47505
\end{gathered}
$$

\section{Acknowledgements}

The author would like to thank the anonymous referee for his/her careful reading and many kind and helpful comments.

The author dedicates this paper with great respect to Professor Katsuhisa Ohno who made excellent contributions to the field of applied probability. We shall always remember him, not only as a scientist but as a friend and for his humanity. He passed away on 8 August 2013 at the age of 72 .

\section{References}

Bruss, F. T. AND Rogers, L. C. G. (1991). Embedding optimal selection problems in a Poisson process. Stoch. Process. Appl. 38, 267-278.

Chow, Y. S., RobBins, H. AND SiEgmund, D. (1971). Great Expectations: The Theory of Optimal Stopping. Houghton Mifflin, Boston, MA.

FERGUSON, T. S. (2006). Optimal Stopping and Applications. Available at http://www.math.ucla.edu/ tom/Stopping/ Contents.html.

Ferguson, T. S., HARDWICK, J. P. AND TAMAKI, M. (1992). Maximizing the duration of owning a relatively best object. In Strategies for Sequential Search and Selection in Real Time (Contemp. Math. 125), American Mathematical Society, Providence, RI, pp. 37-57.

Gilbert, J. P. AND Mosteller, F. (1966). Recognizing the maximum of a sequence. J. Amer. Statist. Assoc. $61,35-73$.

Gnedin, A. V. (1996). On the full information best-choice problem. J. Appl. Prob. 33, 678-687.

Gnedin, A. V. (2004). Best choice from the planar Poisson process. Stoch. Process. Appl. 111, 317-354.

GNedin, A. V. (2005). Objectives in the best-choice problems. Sequential Anal. 24, 177-188.

Kurushima, A. AND ANo, K. (2009). Maximizing the expected duration of owning a relatively best object in a Poisson process with rankable observations. J. Appl. Prob. 46, 402-414.

Mazalov, V. V. ANd TAmaKi, M. (2006). An explicit formula for the optimal gain in the full-information problem of owning a relatively best object. J. Appl. Prob. 43, 87-101.

Pearce, C. E. M., Szajowski, K. AND TAMAKI, M. (2012). Duration problem with multiple exchanges. Numer. Algebra Control Optimization 2, 333-355.

PoROsIŃsKI, Z. (1987). The full-information best choice problem with a random number of observations. Stoch. Process. Appl. 24, 293-307.

SAKaGUCHI, M. (1973). A note on the dowry problem. Rep. Statist. Appl. Res. Un. Japan. Sci. Eng. 20, 11-17.

SAMUEL-CAHN, E. (1996). Optimal stopping with random horizon with application to the full-information best-choice problem with random freeze. J. Amer. Statist. Assoc. 91, 357-364.

Samuels, S. M. (1982). Exact solutions for the full information best choice problem. Tech. Rep. 82-17, Department of Statistics, Purdue University.

Samuels, S. M. (2004). Why do these quite different best-choice problems have the same solutions? Adv. Appl. Prob. 36, 398-416.

TAMAKI, M. (2009). Optimal choice of the best available applicant in full-information models. J. Appl. Prob. 46, 1086-1099.

TAMaKI, M. (2010). Sum the multiplicative odds to one and stop. J. Appl. Prob. 47, 761-777. 